Cases (biochemical failure) were matched with controls (no biochemical failure) for clinical stage, Gleason score, prostate-specific antigen level, age, race, and androgen deprivation status. A total of 56 pairs were available for analysis. Median follow-up time between cases and controls was comparable (58.5 and 53.5 months, respectively). The number of patients with PNI did not differ significantly between the two groups. Although there was a slight trend towards increased biochemical failure in patients with PNI, Kaplan-Meier analysis revealed that the difference between PNIpositive and $\mathrm{PNI}$-negative patients was not statistically significant $(P=0.4014)$.

Although this study was limited by sample size, the authors conclude that the data are consistent with other reports in the literature that PNI does not have prognostic value in patients with prostate cancer. They suggest that post-therapy external beam radiation for patients with $\mathrm{PNI}$ is, therefore, unnecessary.

Original article Weight CJ et al. (2006) Perineural invasion on prostate needle biopsy does not predict biochemical failure following brachytherapy for prostate cancer. Int $J$ Radiat Oncol Biol Phys 65: 347-350

\section{Low doses of Z-100 are more effective than high doses when combined with radiotherapy}

Radiotherapy alone has limited efficacy in treating patients with advanced cervical cancer; however, radiotherapy might be enhanced by concomitant administration of immunotherapeutic agents. Previous findings indicate that the immunomodulatory drug Z-100 (Zeria Pharmaceutical Co., Ltd, Tokyo, Japan) is most effective as an adjunctive therapy when used at high doses, but a randomized phase III trial by Noda et al. suggests otherwise.

Between June 1995 and March 1999, 221 patients with locally advanced cervical cancer were enrolled in the study; follow-up continued until September 2002. Patients were randomized to low-dose $(0.2 \mu \mathrm{g} / 2 \mathrm{ml} /$ ampoule; $n=109)$ or high-dose $(40 \mu \mathrm{g} / 2 \mathrm{ml} / \mathrm{ampoule} ; n=108)$ Z-100, administered subcutaneously twice weekly, in combination with radiotherapy (50$55 \mathrm{~Gy})$. The response rate was not significantly different between the two groups. Five-year survival was significantly better in the low-dose than in the high-dose group (58.2\% vs $41.5 \%$;
$P=0.039$ ), with the largest difference observed in patients with a higher parametrial invasion. Progression-free survival was also significantly improved in patients receiving the low-dose Z-100 $(P=0.048)$. Survival in the higher-dose group was similar to that in previous studies with radiotherapy alone.

The authors conclude that, when used over a long period, Z-100 improves survival at low doses, but that this survival advantage is lost when the drug is administered at higher doses. They highlight the need for determining optimum doses of immunomodulators and other agents for maximum survival benefit, using placebo controls and high and low doses; a further placebo-controlled, randomized trial is therefore underway.

Original article Noda K et al. (2006) Phase III double-blind randomized trial of radiation therapy for stage IIIb cervical cancer in combination with low- or high-dose Z-100: treatment with immunomodulator, more is not better. Gynecol Oncol 101: 455-463

\section{Reduced lymphoma risk in statin users}

Statins are used to treat hypercholesterolemia and prevent ischemic heart disease. Some studies indicate that these drugs might be carcinogenic, but others indicate that statins reduce the risk of lymphoma and other cancers. Fortuny et al. assessed data from a case-control study to investigate the relationship between chronic statin use and lymphoma risk.

The study included data from 2,362 patients with lymphoma and 2,465 controls $(1,046$ population-based controls and 1,419 hospitalbased controls). Hospital-based controls were classed as group 1 (high probability of statin use; $n=259$ ) or group 2 (probability of statin use similar to that of the general population; $n=1,160$ ).

Statins were used by $3.1 \%$ of patients with lymphoma and $5.4 \%$ of controls $(5.2 \%$ of population controls, $11.6 \%$ of group 1 hospitalbased controls, and $4.3 \%$ of group 2 hospitalbased controls). Risk of lymphoma was lower in patients regularly using statins than in those who had never used statins (odds ratio 0.61 excluding group 1 patients; odds ratio 0.55 including group 1 patients), but duration of statin use did not affect lymphoma risk. Other lipid-lowering drugs were not associated with reduced lymphoma risk. Nonsteroidal anti-inflammatory 